\title{
A Semi Blind Channel Estimation Method BASED ON HYBRID NEURAL NETWORKS FOR UPLINK LTE-A
}

\author{
Lassaad SMIRANI ${ }^{1}$, ihane BOULAHIA ${ }^{2}$ and Ridha BOUALLEGUE ${ }^{3}$ \\ ${ }^{1}$ InnoV'COM Lab, University of Carthage,Tunisia \\ E-learning Deanship \& Distance Learning -Umm Al-Qura University- Makkah KSA \\ ${ }^{2}$ Faculty of social sciences Umm Al-Qura University- Makkah Almoukarramah KSA \\ ${ }^{3}$ Innovation of communication and cooperative mobiles, InnoV'COM Lab, University of \\ Carthage,Tunisia
}

\begin{abstract}
The paper describes how to improve channel estimation in Single Carrier Frequency Division Multiple Access (SC-FDMA) system, using a Hybrid Artificial Neural Networks (HANN). The $3^{\text {rd }}$ Generation Partnership Project (3GPP) standards for uplink Long Term Evolution Advanced (LTE-A) uses pilot based channel estimation technique. This kind of channel estimation method suffers from a considerable loss ofbitrate due to pilot insertion; all data frame sent contains reference signal. The HANN converts data aided channel estimator to semi blind channel estimator. To increase convergence speed, HANN uses some channel propagation Fuzzy Rules to initialize Neural Network parameters before learning instead of a random initialization, so its learning phase ismore rapidly compared to classic ANN.HANN allows more bandwidth efficient and less complexity. Simulation results show that HANN has better computational efficiency than the Minimum Mean Square Error (MMSE) estimator and has faster convergence than classic Neural Networks estimators.
\end{abstract}

\section{KEYWORDS}

Channel Estimation, SC-FDMA, ANN, Fuzzy Logic, LTE-A

\section{INTRODUCTION}

Orthogonal Frequency Division Multiplexing (OFDM) is an access technique proposed as a solution thanks to its multicarrier system, its high data rate and its robustness against Inter Symbol Interference (ISI) and frequency selective fading. Propagation conditions are highly variable and depend on the environment.The transmitted signal encounters different physical mechanisms witch occur during transmission: Reflection, diffraction and scattering. The propagation will have many losses due to the distance and the attenuation caused by obstacles.The received signal is composed of a superposition of delayed versions of the transmitted signal and attenuated. The received signal is a combination of paths whose amplitudes, phase shifts, andDoppler delays differ.The receiver must beprovided with channel estimator which contains the knowledge of Channel Impulse Response (CIR).

Channel estimationmethods in the OFDM systems context have been widely studied[15]. They belong to three families: Blind channel estimation, supervised channel estimation and channel estimation with decision feedback. The most effective techniques offer a good compromise between performance and complexity; in general they are based on the insertion of known symbols, called pilot, in the data frame. Also, there are many types of pilot arrangement method:

DOI : $10.5121 /$ ijwmn.2016.8305 
the estimators using the block arrangement can be based on the algorithm of least squares (LS) or the algorithm Minimum Mean Square Error (MMSE)... The other estimators use the comb arrangement and they caninclude the previous algorithms with interpolation.We can also find a third typeof pilot arrangement method which is a combination between the two methodscited previously.

The Artificial Neural Networks (ANN) has been applied in many fields.The performance of the OFDM system can be improved by using an ANN to perform channel estimationThere are many studies in which ANN algorithms are used for channel estimation [3][4][5][6][7][8]. Even though there are only a few estimation algorithms evaluated for SC-FDMA by using ANN for channel estimation [4][5][8][9],Although, there is no study in literature based on hybrid neural network for channel estimation.

In this paper we present a channel estimation method using a special neural network for uplink LTE-A. Thisstudy combines neural networks with Fuzzy Logic method, to improve the network efficiency by reducing the insertion of reference signals to estimate the channel frequency response.The rest of this paper is organized as follows: Section II provides the brief idea about the OFDM and SC-FDMA Systems Model. Section III describes the multipath mobile radio propagation channel model, in section IV classic channel estimation methods; Least Square (LS) [2], MMSE [3] and estimation with classic artificial neural networks are presented. The section V presents the HANN and its advantages. The proposed channel estimation technique by HANN is introduced in section VI. In section VIIsimulation results and a comparative study with the classic estimation methods is presented. Finally, Section VIII concludesthis paper.

\section{OFDM AND SC-FDMA SYSTEM MODEL}

The OFDM has been chosen as a solution for many new transmission systems. The choice of OFDM is mainly due to the high flow rate offered by this solution, the flexibility to adapt with channel propagation, the simplicity of the implementation, and the imminent against the interference.LTE-A is based on OFDMA air interface transmission scheme in downlink and SCFDMA scheme in uplink. SC-FDMA belongs to the multiple access family. It is similar to OFDMA system except that the first technique presents FFT as a first operation in the transmitter,especially to reduce Peak to Average Power Ratio (PAPR). Like any system mono carrier, the SC-FDMA uses a simple modulation with equalization in the frequency domain (SC/FDE) to adapt the multi-user access. Its block diagram is given by Figure 1.

We are interested in this paper on uplink, so we will present the SC-FDMA system model which is shown in Figure 1. The SC-FDMA can be viewed as an OFDMA for which we just add a Fast Fourier Transform before proceeding to the implementation of the OFDMA modulation. The application of the FFT transforms the data symbols from the time domain to the frequency domain.

SC-FDMAmodulationcan be divided into threemain parts which are: FFT modulator,module of frequency resources allocation, and finallyanIFFT modulator. The FFT modulatortransforms thesource symbolsfrom time domain to frequency domain. The module of frequency resources allocationwillallocate frequency symbol obtainedonallsubcarriers of thesystemaccording totwopossiblemodescalled thelocalized modeanddistributed mode. The IFFTmodule thenallows the conversion of the frequencysymbolsin time domain. In the uplink, data is mapped onto a signal constellation that can be QPSK, 16QAM, or 64QAM depending on channel quality.

The output result of the FFT block is a discrete frequency domain representation of the QPSK/QAM symbol sequence. The discrete Fourier terms at the output of the FFT block are then mapped to subcarriers before being converted back into the time domain (IFFT). 


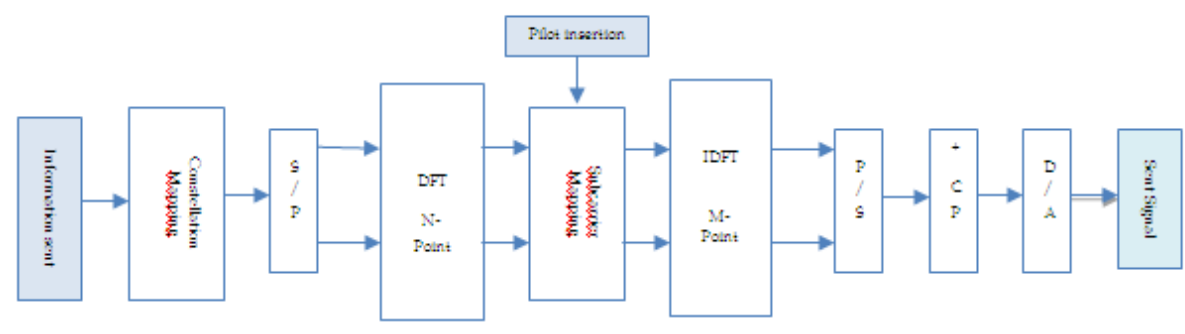

Figure1. A block-diagram of an uplink SC-FDMA transmitter

The final step is appending a CP. It is interesting to note that while the SC-FDMA signal has a lower PAPR in the time domain, individual subcarrier amplitudes can vary more in the frequency domain than a comparable OFDM signal

\section{Channel Estimation}

Channel estimation is one of the most significant technologies in the orthogonal OFDM system and its accuracy will influence the performance of the whole system directly. In LTE-A there is a packet dedicated reference signals in each slot SC-FDMA signal. The reference signals constituting an SC-FDMA packet are located on all subcarriers of the system. This allows an estimation of all subcarriers by a simple inversion of symbols pilots. In LTE-A standards the reference signals are inserted once per seven time slots, the performance of estimation depends on channel speed variation. The signal obtained at the receiver is usually not identical to the transmitted signal. It may suffer attenuations and distortions even disappearing of certain frequency components of the signal. Other spurious signals can be added to the original signal during transmission in addition to noise.

$$
I(t)=\sqrt{\frac{2 W}{T}} \cos \left(2 \pi f t+\varphi_{i}\right)
$$

Where $I(t)$ is the signaling waveforms produced by the baseband multiple shift keying modulator after taking binary sequence.

Wis the energy of $I(t), T$ is the symbol duration, $f$ is the frequency of the carrier and we have $f=$ $\frac{\omega}{2 \pi}$.

$\varphi_{i}$ is the phase and we have $\varphi_{i}=\frac{2 \pi}{N}, \mathrm{~N}$ is the alphabet size.

Then we have, after developing the expression of $I(t)$, two terms in phase basis and quadrature basis.Assume that we have U users, $0 \leq u \leq U-1$ in the LTE uplink connection, for each user, the SC-FDMA transmitter will make the operations listed above and described in figure1. The first operation done by the SC-FDMA transmitter is grouping the modulation symbols into blocks $S_{N}$, Where $S_{N} \in\left\{S_{0}, S_{1}, \ldots \ldots . S_{N-1}\right\}$. So we will have N symbols for each block.

Where DFT is the Discrete Fourier Transform, it converts the symbols into the list of coefficients of acomplex sinusoid combination, ordered by their frequencies.Its rule also is to convert the input signal from the time domain to the frequency domain as follows: 


$$
\mathrm{N}-\mathrm{DFT}=\left(\begin{array}{ccc}
W_{N}^{0,0} & \cdots & W_{N}^{0, N_{c}-1} \\
\vdots & \ddots & \vdots \\
W_{N}^{N-1 . .0} & \cdots & W_{N}^{N-1, N_{c}-1}
\end{array}\right)
$$

Where $\mathrm{N}$ is the DFT size and

$$
W_{N}^{l, m}=\frac{1}{\sqrt{N}} e^{\frac{-j 2 \pi l m}{N}}
$$

The most important operations made by the transmitter are:

\section{N-point DFT}

$$
D^{u}(k)=\frac{1}{\sqrt{N}} \sum_{n=0}^{N-1} S^{u}(n) e^{\frac{-j 2 \pi n k}{N}}
$$

\section{Distributed or localized subcarriers mapping.}

$$
M_{k}^{u}= \begin{cases}D_{k}^{u} \text { fork }=\nabla_{N}^{u}(k) \\ 0 \quad \text { Otherwise }\end{cases}
$$

\section{M-point IDFT:}

$$
x^{u}(k)=\frac{1}{\sqrt{M}} \sum_{n=0}^{M-1} X^{u}(n) e^{\frac{j 2 \pi n k}{M}}
$$

Input data stream of user $u$ is mapped to quadrature-amplitude modulation (QAM) symbols.

Block of $S$ symbols is then converted to the frequency domain using $N$-point DFT. Then, $N$ output samples are mapped to the user's subcarriers by distributed or localized subcarrier mapping.

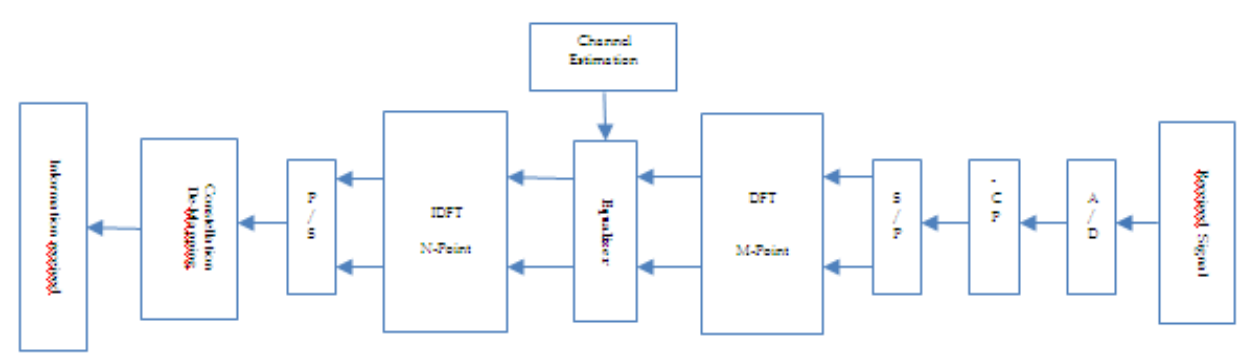

Figure 2. A block-diagram of an uplink SC-FDMA receiver

Distributed mapping occupies a comb-shaped spectrum whereas localized mapping occupies a continuous spectrum.

To achieve channel estimation, we insert pilot symbols into data sequences. The modulated symbols and pilots perform N-point DFT to produce a frequency domain representation[5]. After the mapping, the IDFT module output is followed by a cyclic prefix $(\mathrm{CP})$ insertion that completes the digital stage of the signal flow. 


\subsection{Received signal Model}

At the receiver, the first operation made is the synchronization then the receiver has to realize the full inverse operation made by the transmitter, so it discards CP and applies the DFT processing to the rest of samples in order to retrieve the complex constellation symbols transmitted over orthogonal sub-channels. The received signal $\mathrm{y}(\mathrm{n})$ is given as:

$$
\mathrm{y}(\mathrm{n})=\sum_{\mathrm{u}=0}^{\mathrm{U}-1} \sum_{\mathrm{l}=0}^{\mathrm{L}-1} \mathrm{~h}^{\mathrm{u}}(\mathrm{n}, \mathrm{l}) \mathrm{x}^{\mathrm{u}}(\mathrm{n}-\mathrm{l})+\mathrm{Z}^{\mathrm{u}}(\mathrm{n})
$$

The DFT output at $\mathrm{p}^{\text {th }}$ subcarrier for user $\mathrm{u}$ is given as:

$$
Y^{u}=G^{u}(p, p) X^{u}(p)+\sum_{f=o, f \neq p}^{f \in \nabla^{u}} G^{u}(p, f) X^{u}(f)+Z^{u}(p)
$$

The Channel Transfer Impulse is given as:

$$
\mathrm{G}^{\mathrm{u}}(\mathrm{p}, \mathrm{f})=\frac{1}{\mathrm{~N}} \sum_{\mathrm{n}=0}^{\mathrm{N}-1} \sum_{\mathrm{l}=0}^{\mathrm{L}-1} \mathrm{~h}^{\mathrm{u}}(\mathrm{n}, \mathrm{l}) \mathrm{e}^{\left(-\mathrm{j} \frac{2 \pi \mathrm{fl}}{\mathrm{N}}\right)} \mathrm{e}^{\left(\mathrm{j} \frac{2 \pi(\mathrm{f}-\mathrm{p})}{\mathrm{N}}\right)}
$$

\subsection{Classic Estimators}

In this section we will study two classic estimators before introducing the HANN. The wellknownestimator in literature is Least Square Estimator (LSE)[3]. LSE is simple and easy to apply, its major drawback that it doesn't take in consideration correlation between two adjacent symbols and therefore the symbols are estimated separately one by one. A second disadvantage of this method is its sensitivity to noise and selectivity [3].

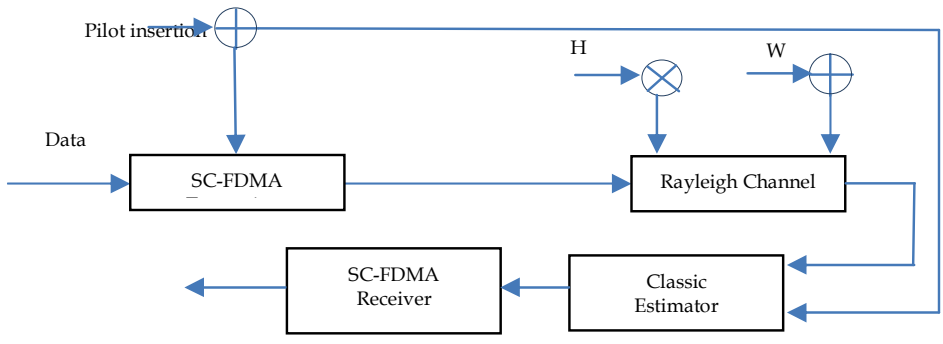

Figure 3. Classic ANN Estimator Model

The LSE is the most used for its known simplicity but its performance is limited as we have explained. After LSE, we find in literature Minimum Mean Square Estimator (MMSE)[4]. The MMSE has better performance than LSE but it has a high complexity. We also find in the literature other estimators based on LSE or MMSE, on which changes were made to make a compromise between complexity and performance [2].

\subsection{The Least Square Estimator LSE}

The least square criterion aims to minimize cost function F. F is defined as the square norm of an error vector. The error vector is obtained by calculating the difference between the vector $\mathrm{R}$ of the received signal and the product of the transmitted signal vector $\mathrm{E}$ with a diagonal matrix whose coefficients are M assumed to be optimal. The minimum squared error for classic LSE will be given by: 


$$
\begin{array}{r}
\operatorname{MMSE}_{\mathrm{LS}}=\frac{1}{\mathrm{M}} \mathrm{E}\left\{\operatorname{tr}\left(\mathrm{WE}^{-1}\left(\mathrm{WE}^{-1}\right)^{\mathrm{H}}\right)\right\} \\
=\frac{1}{\mathrm{M}} \sum_{\mathrm{i}=0}^{\mathrm{M}-1} \frac{\mathrm{E}\left\{\mathrm{W}_{\mathrm{i}} \mathrm{W}_{\mathrm{i}}^{*}\right\}}{\mathrm{P}}=\frac{\boldsymbol{\sigma}^{2}}{\mathrm{P}}
\end{array}
$$

The minimum mean squared error of an LS estimator is equivalent to the inverse of the SNR. In these conditions, the interpolation method must be taken into consideration.

\subsection{The Minimum Mean Square Error estimator: MMSE}

This method takes into account the correlation of the channel, that makes it more precise than the first method. A 2D Wiener filter exploiting time and frequency correlations filter is optimal in the sense of the mean squared error. This MMSE estimator has two major difficulties:The first is the implementation complexity, since two independent filters are applied successively. The second challenge is to calculate from the knowledge of certain properties of sub-channels (Doppler frequency, maximum spread sub-channels). It is obvious that the performance of the MMSE estimator is much better than the LSE especially if the signal to noise ratio is low.

$$
\left.\widehat{H}^{L M M S E}=\underline{R}_{H}\left(\underline{R}_{H}+\underline{(E}^{H} \underline{E}\right)^{-1} \sigma^{2} \underline{I}\right)^{-1} \widehat{H}^{L S}
$$

It is clear that channel noise has a significant impact in channel estimator behavior. MMSE is less sensitive to noise than LSE. In the next section we introduce the improvement made by neural networks.

This paper presents a Neuro-Fuzzy solution to achieve an adequate interpolation for LTE-A channel estimation. This interpolation aims to estimate the channel impultionnelle response for all received data symbols by using a few pilot symbols.

\subsection{Artificial Neural Networks (Ann)}

ANN is a branch of artificial intelligence;Neural Networks are an attempt to simulate the brain. These networks are composed with neurons connected to each other by weighting followed by an activation function.

Three minimum layers compose a multilayer feed forward ANN. A layer is a set of neurons placed at the same level. In figure 4, we show the first layer in the input of the ANN, the hidden layer and the output layer. An artificial neuron receives a number of inputs either from original data, or from the output of other neurons in the NN. Each input comes via a connection. Every connection has weight value. These weights correspond to synaptic efficacy in a biological neuron. Each neuron also has a single threshold value. The weighted sum of the inputs is formed, and the threshold subtracted, to compose the activation of the neuron. The activation signal is passed through a transfer function to produce the output of the neuron. ANNs utilized different type of activation functions. Learning is the most important step in neural network methods.

Learning is a dynamic and iterative process to change the occur settings. There are several types of learning method. The two most well-known one are supervised learning and unsupervised learning. In the first one, precise information about the desired output is available and the network learns by presenting peer input/output. During learning, the desired output values are compared to those produced by the network, the resulting error is used for adjusting the connection weights. But when using the unsupervised learning method no information about the desired network 
output is available. Thus, the network handles data presented to it as input and attempts to extract some properties that will form the network outputs.

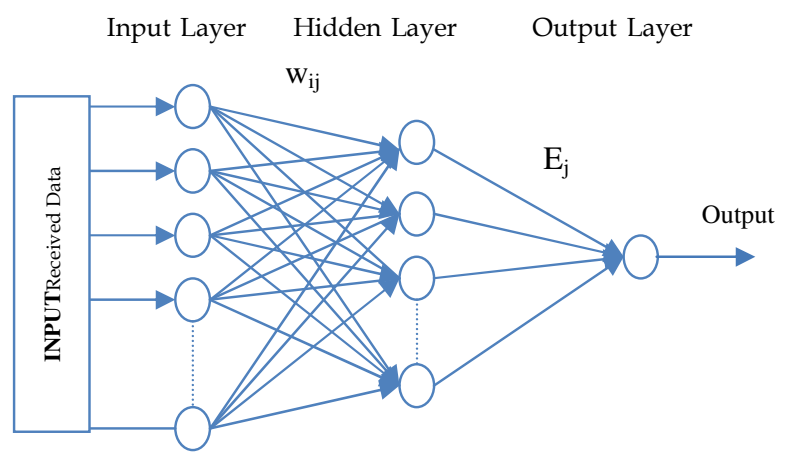

Figure 4.ANN structure

The extraction of these properties depends on the learning rule used in the network.

In our system we have used a modified neural network. The proposed system consists of a conventional neural network to which were added components in order to decrease the complexity and convergence speed. Let us begin by a rapid description of neural network algorithm as shown in figure 4 .

Output of header layer is computed as shown:

$$
N_{i}=\sum_{i=0}^{i=l} E_{i} W_{i, j}
$$

Where $\mathrm{W}_{\mathrm{i}, \mathrm{i}}$ is input to hidden layer weights, $\mathrm{E}_{\mathrm{i}}$ is the input data of Unit $\mathrm{i}$. The output hidden layer is represented as:

$$
O_{l}^{j}=f\left(N_{j}\right)=\frac{1}{1+e^{-N_{j}}}
$$

Where $\mathrm{f}$ is the activation function, the final neural network output can be written as:

$$
N_{i}=\sum_{i=0}^{i=l} O_{l, j} W_{i, j}
$$

We use the back propagation neural network algorithm which is a supervised learning.

It aims to reduce the difference between the network output and desired output values $\mathrm{D}_{\mathrm{i}}$, so we define error function as follow:

$$
\text { Error }=\frac{1}{2} \sum\left(D_{i}-O_{l}^{i}\right)^{2}
$$

\subsection{Approach Description}

To learn a simple ANN, the weights are initialized randomly. However, ANN may take time to converge to the desired value. When ANN is executed, the input variable values are placed in the input units, and then the hidden and output layer units are progressively executed. Each of them calculates its activation value by taking the weighted sum of the outputs of the units in the preceding layer, and subtracting the threshold. The activation value is passed through the 
activation function to produce the output of the neuron. When the entire network has been executed, the outputs of the output layer act as the output of the entire network. There is many categories of ANN like feed-forward neural network or in cascade-forward neural network.

\subsection{Hybrid Artificial Neural Networks :HANN}

Our contribution in this paper is a new neural network called HANN, with rapid convergence and less complexity. The system provides facilities to initialize algorithm before making training, so instead of taking initial weights randomly, we propose to choose the initial values. This choice is made by the symbolic module. The system transfer fuzzy rules from a symbolic module to a connectionist one, and transfer examples in the opposite sense. We start by initializing the neural network. This initialization is made by the insertion rules module using the databases rules. This approach solves two big problems related to artificial neural networks: On one hand this simplifies the choice of the number of units; On the other hand we obtain a good assignment of initial values to the connection weights.

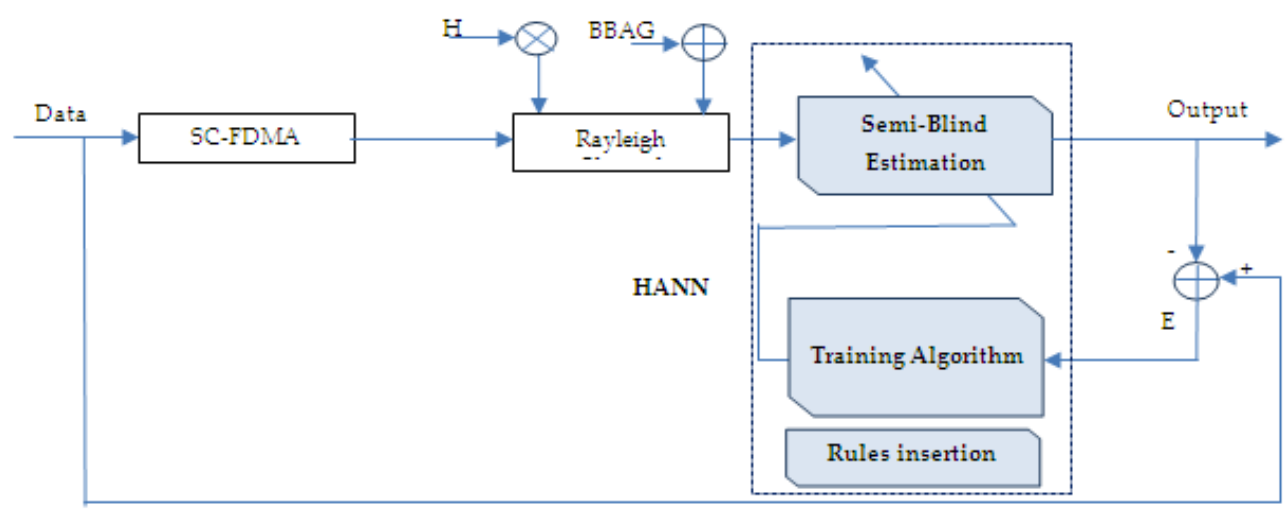

Figure 5. Channel Estimation using HANN

The classic ANN estimator uses the information provided by received reference symbols of sub channels to estimate the total channel frequency response. Two parallel ANN are used, one for real input pilot symbol and the other for imaginary one. In the learning phase, inputs neural network are the received pilot symbols and the target are the sent one. In generalization phase HANN doesn't need to use one inserted pilot by slot.

Table 1. Simulation parameters

\begin{tabular}{|l|l|}
\hline Parameter & Value \\
\hline Number of sub carrier & 512 \\
\hline IFFT and FFT size & 512 \\
\hline Noise Model & AWGN \\
\hline Modulation & 8 QAM and 16 QAM \\
\hline Channel Type & Rayleigh channel \\
\hline Number of symbol & 1000 \\
\hline Guard Type & Cyclic extension \\
\hline
\end{tabular}

HANN is composed of two important modules: Fuzzy Rules Initialization Module and Connectionist Module. We tested HANN by insertion of two fuzzy rules and with insertion of only one symbol pilot in two slots. 
As a classic ANN, the first step is to start training the neural network. The most important step in HANN process is the initialization of neural network weights. This initialization is made by the insertion rules module using the database system rules.

This approach provides HANN to converge rapidly by obtaining a good assignment of initial values to the connection weights.

HANN is initialized by architecture, bias and connections weights. To increase convergence speed, HANN uses some channel propagation rules to initialize Neural Network weights, so its learning phase is more rapidly compared to classic ANN. Moreover the connectionist module is activated in order to train the neural network. This training is based on a set of examples. By using Matlab, data and pilots are sent in SC-FDMA system. The estimator uses transmitted signal with only one pilot by two slots, after passing through AWGN and Flat Rayleigh channel and adding noise. The simulation program of HANN follows the following steps:

(1) Learning parameters to define fuzzy rules

(2) Initialization of HANN weights

(3) Take received signal as an input and transmitted signal as a target

(4) Total error $=$ zero; iterations- $>$ iterations +1

(5) Feed it to input layer units.

(6) Initialize the target output of that ANN.

(7) Calculate the outputs of hidden layer units.

(8) Calculate the outputs of output layer units.

(9) Calculate the error $=$ desired output - actual output, Total_error $->$ Total_error + error.

(10) Calculate delta sigma of output neurons. Then adjust weights between output and hidden layer units.

(11) Calculate delta sigma of hidden layer units. Then adjust weights between hidden and input layer units.

(12) While there are more data in the file, go to step 4

(13) If Threshold error $>=$ Total error then stop, otherwise go to the 3rd steps in this algorithm

In simulations, we compare HANN in SC-FDMA system with LS, MMSE and Classic ANN. The same channel conditions in LTE-A are used on simulation tests (Frequency, Bandwidth). The significant parameters used for simulations are mentioned in Table I.

\section{Simulation RESUlts}

First we prove the contribution added by a classic ANN.

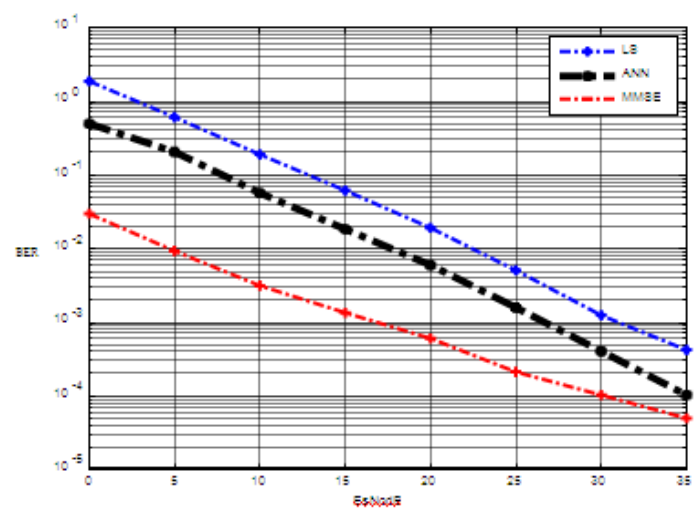

Figure 6. LS, MMSE and ANN simulation Channel Estimation SC-FDMA using 8 QAM 
The figure 6 shows that the performance of the ANN Estimator is better when compared to LS Estimator in terms of Bit Error rate (BER).After, we compare our proposed Neural Networks method to classic one.Simulation results show that our proposed method offers better performance than classic ANN method.

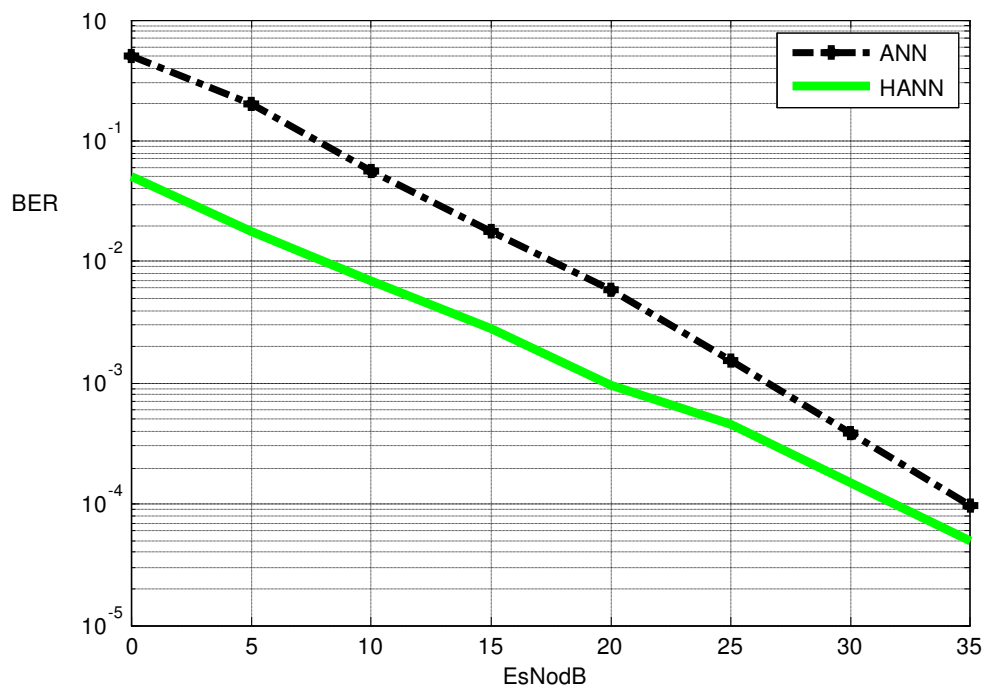

Figure 7. HANN and ANN simulation channel estimation SC-FDMA using 8 QAM

By using Artificial Neural Network, as can be seen from the figure 7, the best performance is obtained through the use of the HANN.

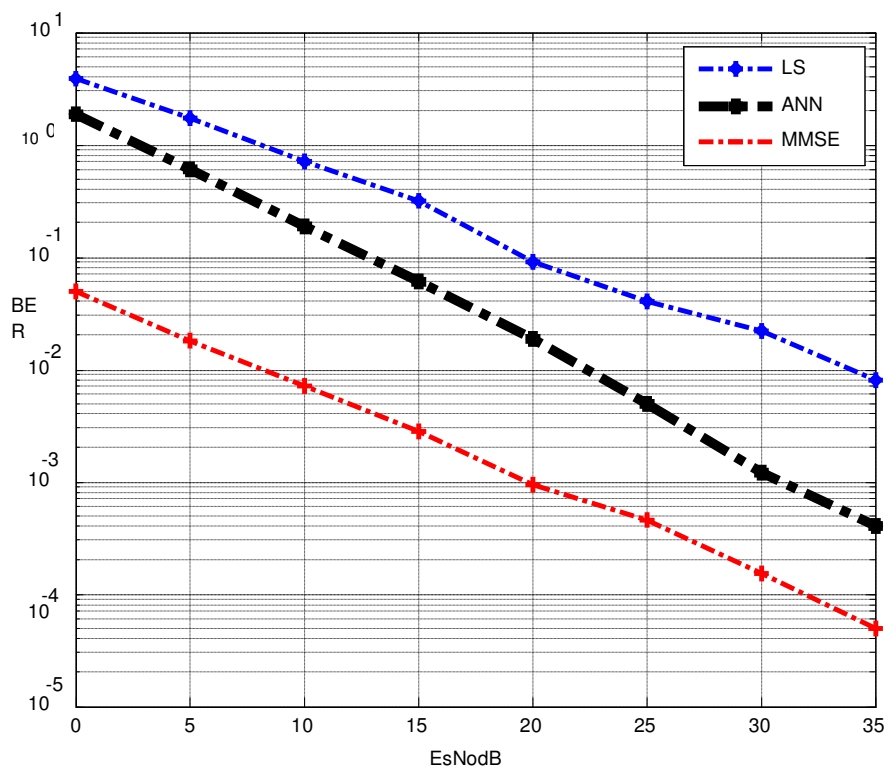

Figure 8 LS, ANN and MMSE simulation channel estimation SC-FDMA using 16 QAM

Simulation results validate the theoretical findings. Only the average of 15 iterations is needed for the block HANN algorithm to converge; but the ANN needs more iteration 
to converge. It is clear that increase of convergence speed of HANN due to use of channel propagation fuzzy rules to initialize Neural Network weights. The Bit Error Rate (BER) performance of the LS, MMSE, ANN and HANN is shown in figure 7 and figure 9, by using 8 QAM and 16 QAM modulations respectively. It is clear that, like all channel estimator, performance depends on modulation scheme.

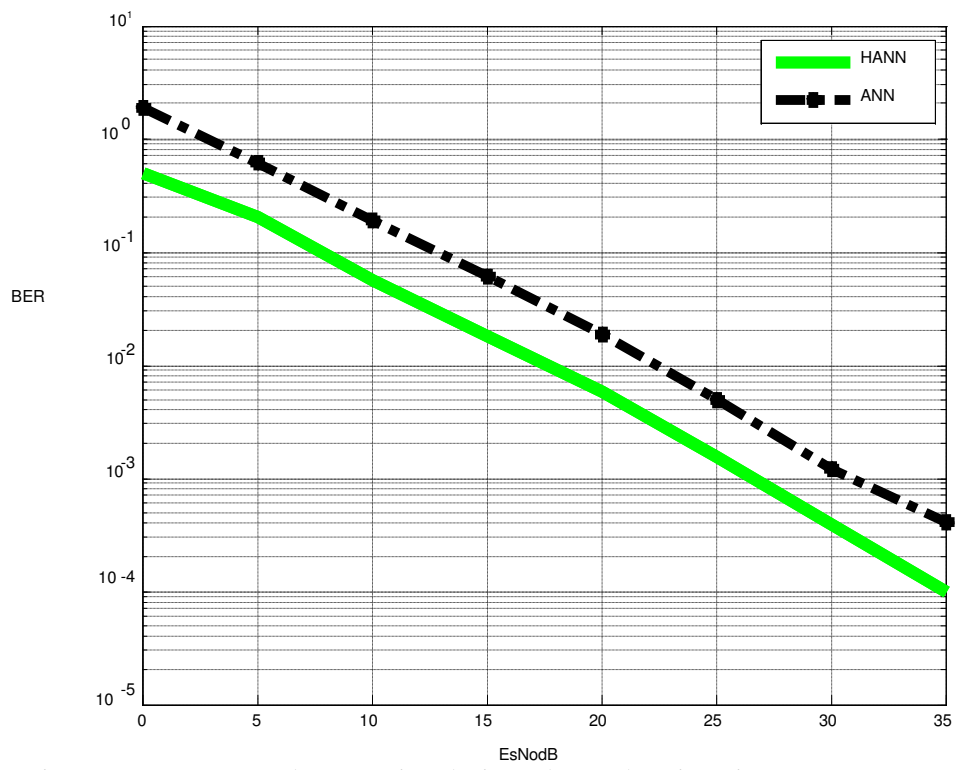

Figure 9. HANN and ANN simulation channel estimation SC-FDMA using 16 QAM

Thanks to its rules insertion function, HANNoffers better results than that of a conventional ANN. The fuzzy rules inserted at the beginning to guide the operation of neural networks learning. Several fuzzy rules can be used for this task. These rules may affect the quality channel variation or the throughput variation.

\section{CONCLUSION}

In this paper we study the channel estimation in SC-FDMA context. Our proposed HANN algorithm contains an insertion fuzzy rules module that gives it the ability to converge rapidly and to operate withfew pilot insertions. Simulation results show that, compared to LS and classic ANN algorithms, HANN presents better performance. Moreover, our proposed algorithm offers two important advantages; The first is the maximum of bandwidth efficiency because of reduction of pilot insertion number. The second, HANN delivers its performance at low complexity than the MMSE algorithm.

\section{REFERENCES}

[1] 3rd Generation Partnership Project, Technical Specification Group Radio Access Network; evolved Universal Terrestrial Radio Access (UTRA): Physical Channels and Modulation layer, pp. 55 - 67, TS 36.211, V8.8.0, 2009.

[2] J. Ketonen, M. Juntti, and J. R. Cavallaro, "Performance complexity comparison of receivers for a mimo-ofdm system," IEEE Transaction on Signal Processing, vol. 58, pp. 3360-3372, 2010.

[3] C. Lim and D. Han, "Robust ls channel estimation with phase rotation for single frequency network in ofdm,” IEEE Transactions on Consumer Electronics, vol. 52, pp. 1173-1178, 2006. 
[4] S. Galih, T.Adiono, and A.Kurniawan, "Low complexity mmse channel estimation by weight matrix elements sampling for downlink ofdma mobile wimax system," International Journal of Computer Science and Network Security b (IJCSNS), vol. 528, pp. 1173-1178, 2010.

[5] B. Karakaya, H. Arslan and H.Ali Cirpan, "Channel Estimation for LTE Uplink in High Doppler Spread", WCNC 2008.

[6] N. K. Ibrahim, R.S.A. Raja Abdullah and M.I. Saripan, "Artificial Neural Network Approach in Radar Target Classification,” Journal of Computer Science, vol. 5, no.1, pp.23-32, ISSN: 1549-3636, Science Publications, 2009.

[7] R. P. Lippmann, “An Introduction to Computing with Neural Nets,"IEEE ASSP Magazine, vol.4, no.2, pp.4-22, April 1987.

[8] Chin-Teng Lin and C.S. George Lee, "Neural Fuzzy System,” Pearson Education Taiwan Ltd, 1996.

[9] NecmiTaspmar, and M. Nuri Seyman, "Back Propagation Neural Network Approach for Channel Estimation in OFDM System," Wireless Communications, Networking and Information Security (WCNIS), 2010 IEEE International Conference on, pp. 265-268, June 2010

[10] T. Cui, and C. TeJlambura, "Channel estimation for OFDM systems based on adaptive radial basis function network," In Proceeding of the IEEE 60th Vehicular Technology Conference, Los Angeles, USA, pp.608-611, September 2004.

[11] Wang, X., Li, X., \& Leung, V. C. (2015). Artificial Intelligence-Based Techniques for Emerging Heterogeneous Network: State of the Arts, Opportunities, and Challenges. IEEE Access, 3, 13791391.

[12] C. Zhang, Z. Wang, Z. Yang, J. Wang, and J. Song, "Frequency Domain Decision Feedback Equalization for Uplink SC-FDMA," IEEE Transactions on Broadcasting, vol. 56, pp. 253-257, June 2010.

[13] W. Younis, A. Sayed, and N. Al-Dhahir, "Efficient adaptive receivers for joint equalization and interference cancellation in multiuser space-time block-coded systems," IEEE Transactions on Signal Processing, vol. 51, pp. 2849-2862, Nov. 2003.

[14] Yong Soo Cho, Jaekwon Kim, Won Young Yang, and Chung G. Kang, "MIMO-OFDM Wireless Communications with MATLAB," John Wiley \& Sons (Asia) Pte Ltd, 2 Clementi Loop, \#0201,Singapore 129809, 2010.

[15] Cheng-Hung Chen; Cheng-Jian Lin; Chin-Teng Lin, "A Recurrent Functional-Link-Based Neural Fuzzy System and Its Applications," Computational Intelligence in Image and Signal Processing, 2007. CIISP 2007. IEEE Symposium on, vol., no.,pp.415,420, 1-5 April 2007.

[16] Patra, J.C.; Pal, R.N., "Functional link artificial neural network-based adaptive channel equalization of nonlinear channels with QAM signal," Systems, Man and Cybernetics, 1995. Intelligent Systems for the 21st Century., IEEE International Conference on , vol.3, no., pp.2081,2086 vol.3, 22-25 Oct 1995.

[17] C. T. Lin and C. S. G. Lee, Neural Fuzzy System: A Neuro-Fuzzy Synergism to intelligent systems, NJ: Prentice Hall PTR, 1996.

[18] J. S. R. Jang, S. C. Tsai and MizutaniEiji, Neural-fuzzy and soft computing?a computational approach to learning and machine intelligence, NJ: Prentice Hall, 1997.

[19] SMIRANI L., BOULAHIA J. and BOUALLEGUE R. "Artificial Neural Network Interpolation for SC-FDMA Channel Estimation" in 2nd International Conference and Business Expo on Wireless \& Telecommunication 2016 Dubai UAE.

[20] SMIRANI L. and BOULAHIA J.Neural rule extraction: more precision in learning.WSEAS International Conference on Neural Network LISBON 2014.

[21] SMIRANI L. and BOUALLEGUE R. "An Uplink LTE-A Channel Estimation Method Based On Connexionist System”Energy and Manufacturing Engineering (ICAEME'2015 Jan. 7-8, 2015 at Dubai (UAE).

[22] SMIRANI L and BOULAHIA J . A New Channel Estimation Method for LTE-Advanced Uplink . ICMCE 2015 : XIII International Conference on Materials and Chemical Engineering. January 26 27, 2015 . JEDDAH.

[23] BOULAHIA J. and SMIRANI L. Experiments of Neuro Symbolic Hybrid Learning System With Incomplete Data" International Conference on Recent Advances in Engineering and Technology (ICRAET-2015), Feb 2015 Istambul, Turkey. 